Annales UMCS

Informatica

Lublin-Polonia

Sectio AI

http://www.annales.umcs.lublin.pl/

\title{
Load balancing of communication channels with the use of routing protocols
}

\author{
Franciszek Grabowski $^{1 *}$, Marek Bolanowski ${ }^{2 \dagger}$, Andrzej Paszkiewicz $^{3 \ddagger}$ \\ ${ }^{1}$ Rzeszów University of Technology, Faculty of Electrical and Computer Engineering, \\ Department of Distributed Systems, \\ al. Powstańców Warszawy 12, 35-959 Rzeszów, Poland
}

\begin{abstract}
In the article the authors propose a method for load-balancing of network resources for the case which uses a routing protocols. In the first part of the article the authors present currently used algorithms for load balancing and possibilities of their modification. Through the introduction of additional hardware components for each node: the agent and the probe; it is possible to monitor and control the current system performance. The whole analyzed network is treated as a complex system. This allows to eliminate overloading of route nodes (through ongoing analysis of the optimal operating point for a given node). Load balancing can be achieved using a modified mechanism of ECMP. The proposed approach allows for dynamic adjustment of load to network resources and thus effectively to balance network traffic.
\end{abstract}

\section{Introduction}

Network traffic handling and, in particular, meeting its specific quality requirements is a difficult and particularly important task. Currently in such environment as Ethernet to carry out these tasks there are used specialized protocols which try, in a limited way, to introduce artificially the classification and prioritizing of the traffic at the micro level. It seems appropriate to apply a holistic approach to unify the load of network resources, which may in turn lead to more efficient traffic handling and create the possibility to affect the quality of the transmission. A form of mechanisms of conscious

*fgrab@prz.edu.pl

${ }^{\dagger}$ marekb@prz.edu.pl

‡andrzejp@prz.edu.pl 
traffic control can be demonstrated in operator networks where we can talk about traffic engineering [1]. With the use of the Protocols MPLS, ATM or even Frame Relay $[1,2,3,4]$ components of the system can be loaded by the traffic evenly and thus give real traffic handling capabilities with the specified parameters. Unfortunately, the operator networks represent a small fragment of the whole transmission path of user's data. User's data is also transmitted by the client and corporate networks which, for needs of this article we call "networks routable in the third layer".

\section{Route selection in the classical routing protocols}

Routable networks of the third layer. Let us see how elements of a simple network (Fig. 1) are used by the traffic directed using standard routing protocols [5, $6,7]$.

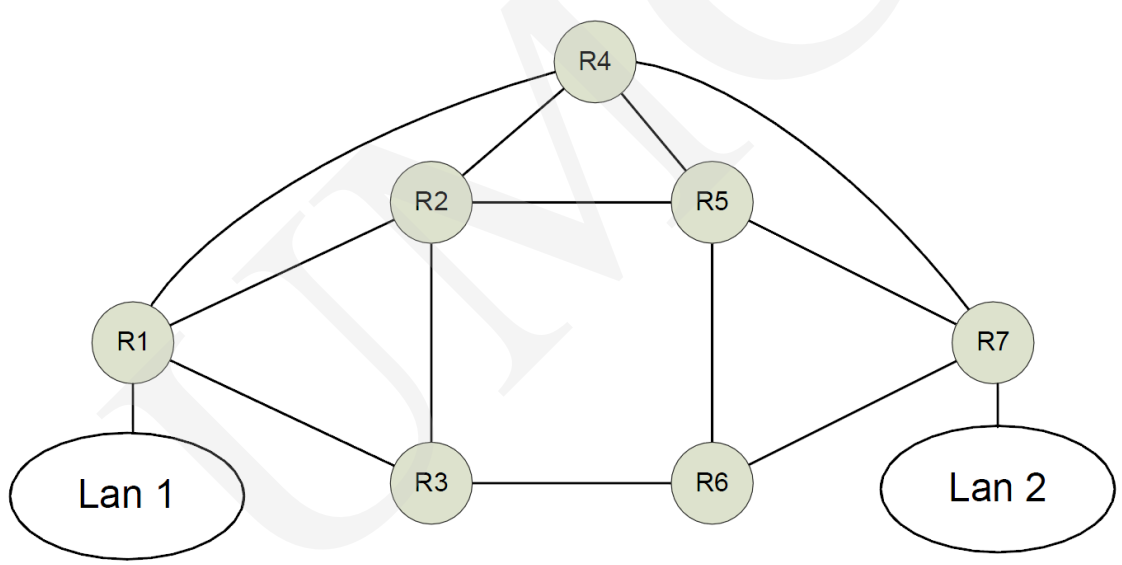

Fig. 1. Routed network.

Case 1: If we consider protocols of the distance vector type, it turns out that the most advantageous route between the networks LAN1 and LAN2 is the route R1/R4/R7.

Case 2: If we consider protocols of the link state type, it may turn out that the most advantageous combination is the routes $\mathrm{R} 1 / \mathrm{R} 2 / \mathrm{R} 5 / \mathrm{R} 7$ and $\mathrm{R} 1 / \mathrm{R} 3 / \mathrm{R} 6 / \mathrm{R} 7$. Of course, in this case we deal with equivalent routes so as to balance the flows between networks we can use, for example, the ECMP [8] protocol.

For both 1 and 2 cases, there are always network resources on the route between LAN1 and LAN2, which could be used as elements to handle some of the tasks of communication between networks. At present, the mechanism which can perform such a task seems to be the Policy Based Routing, which, depending on the type of data (condition analysis possible in all layers of the ISO/OSI) can direct the traffic on different paths. The problem is that this is a static mechanism, which is used to protect the network, rather than for load balancing. 
The purpose of this article is to develop a mechanism that, based on the current analysis of the load of network resources (transmission channels, devices), will change the routing tables to balance the load of individual network elements and lead to its uniform load. For this purpose, to the classic structure of the network, you need to enter the agents, which will analyze the flows and will control (autonomously or as a group) the network devices using conventional mechanisms such as PBR or the modification of routing tables.

\section{$3 \quad$ Load balancing using routing protocols}

System elements. From the network operation point of view, it seems advisable to define the concept of resources. We can select two fundamental resources of a computer network: a node which consists of input and output ports and the processing elements: a communication channel which transmits data.

For the communication channel the optimal operating point is $100 \%$ saturation of the channel. The transmission of layers 1 and 2 is so formed that a traffic in the channel does not disturb character and is statistically homogeneous. In modern cable transmission channels operating in a full duplex mode of a point to point character there can be no rival access that would cause possible channel overload. Also the output port of the network device makes sure not to overload the channel. From our point of view, particularly interesting seems to be the node - this is a key resource affecting the degradation of parameters describing network traffic. The node works mostly under mismatch conditions and is permanently overloaded with communication tasks. The critical organization of each node can lead to degradation of the efficiency of the whole communication system, and of all the communication paths in the system.

At present the most popular mechanism for load balancing involves giving priority to individual interfaces, and correlating appropriate routing paths with these parameters.

Current traffic management is completely independent of the current traffic load. Statically set parameters P1 to P3 define the whole mechanism of traffic engineering and preclude its effective control.

In the proposed approach, new elements will be introduced to the network: probe $\mathrm{S}$ and control agents $\mathrm{A}$. The task of probes $\mathrm{S}$ is up to date examination of communication channels load (through the analysis of output ports) and report this load to the agent

A. The agent has the following tasks:

1. It analyses the interfaces load and on this basis determines the optimal resources usage (optimal working point).

2. After determining the optimal operating point for each resource agent, to force resources to achieve a balance, it runs the following actions:

a. With the use of the PBR mechanism it moves a part of communication tasks from the overloaded route to the underloaded route.

b. Changes the routing paths in the subordinate routers 


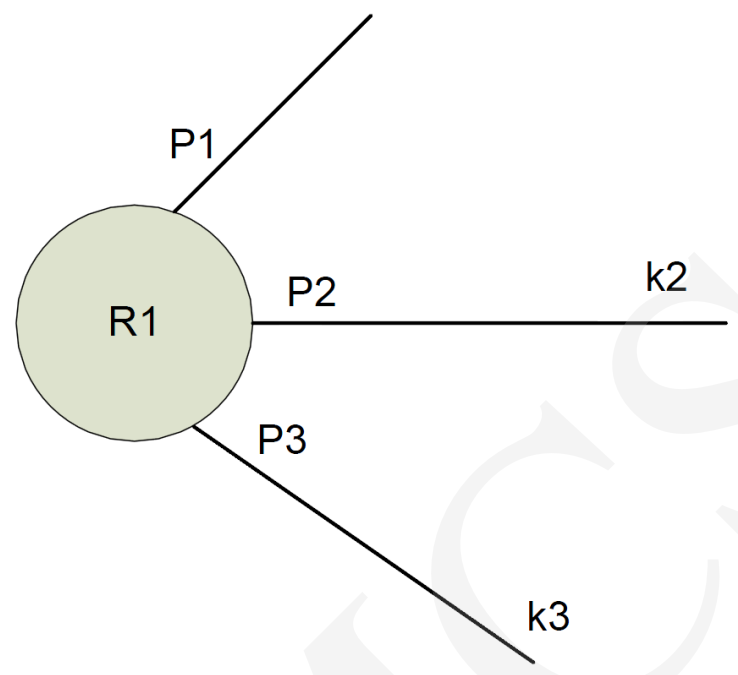

Fig. 2. Present state of traffic prioritisation.

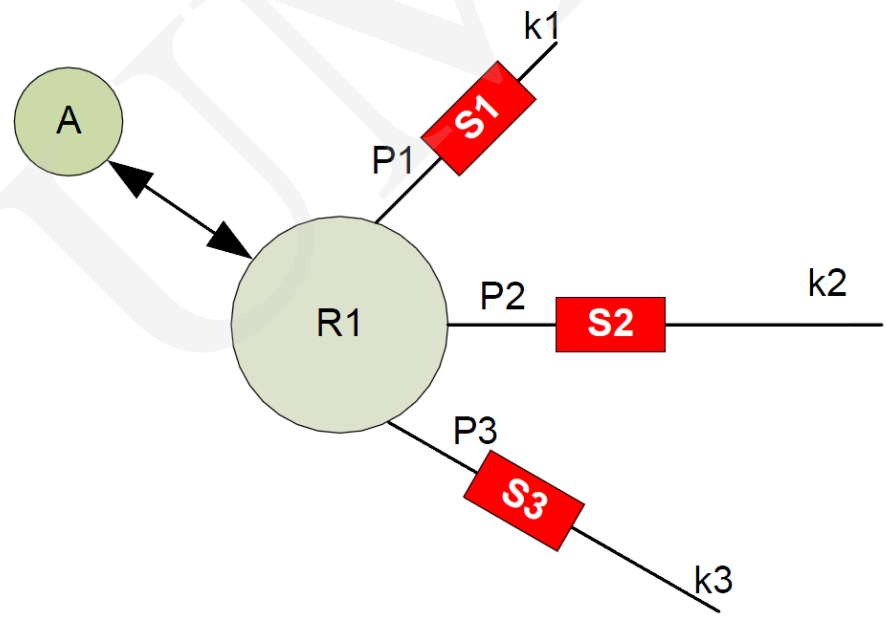

Fig. 3. Proposed expansion of the system.

3. As a result of these actions we obtain load balancing within the routing paths for the same networks through their proportional rather than uniform loading. This is shown in Fig. 4.

We can mark out two types of balancing: within the routes and within the flow of routes. The first type of load balancing is shown by the flows P4 and P5. Paths of routing for unknown networks treat the considered network as one of the transit networks. Despite the common source and destination (R1/R 7) they load other 


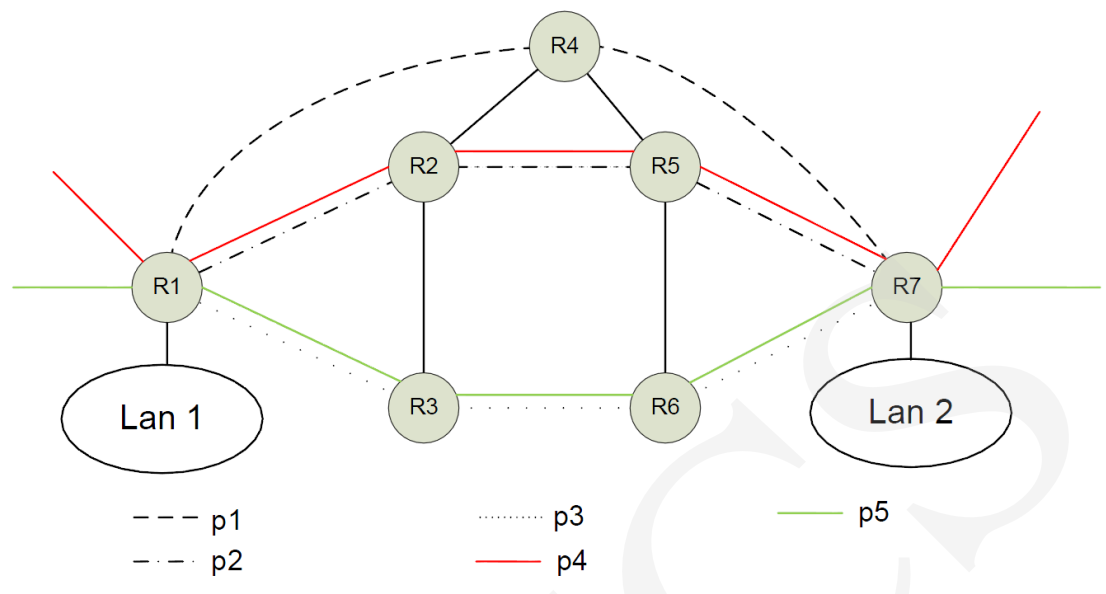

Fig. 4. Flows p1 to p5 - distribution over the network.

intermediate elements of the network. Such balancing may be implemented using a change of entry for the given data flows in the routing table of the router R1. This mechanism is very simple to implement. It differs, however, from the currently used mechanisms, because the routes $\mathrm{R} 1 / \mathrm{R} 2 / \mathrm{R} 5 / \mathrm{R} 7$ and $\mathrm{R} 1 / \mathrm{R} 3 / \mathrm{R} 6 / \mathrm{R} 7$ can have equal or different costs from the routing protocol point of view and can be loaded with flows unevenly. The second type of balancing analyzes the traffic within a single route, separating from it the flows and on this basis divides a single flow into components (e. g. due to the source address, DSCP, TOS, UDP, TCP) and directs the traffic to different routes using the PBR mechanism. An example of such balancing will be the flows p1, p2, p3 which are parts of one route between the networks Lan1 and Lan2.

\section{Load balancing using the routing protocols}

Taking into account the previous considerations, it is clear that the choice of data transmission routes based on commonly accepted criteria in the available routing protocols, can meet the requirements of only homogeneous and not overloaded systems. In order to ensure effective load balancing, we propose the following solutions. Suppose that on the route between the source and the destination there is a complex system. This system usually has a heterogeneous nature, and the processes occurring therein are characterized by periodic or aperiodic explosiveness. From this perspective, the cost parameters with fixed values do not reflect the actual dynamics of the load of such a system. The proposed solution can be based on already existing and widely implemented routing solutions having their own topology base and routing tables. In addition, attention should be paid to the fact that the current routing protocols support the equivalent transmission links, using in this case a simple load balancing. 
Let us introduce the following markings: $K$ means the available resources, $N$ refers to a set of data transmission tasks, $P=\left\{P_{i}: i=1, \ldots, I\right\}$ defines a set of data transmission paths, $N_{P_{i}}$ means the transmission on the $i-$ th path.

Accepting that each network device (e. g. router) which is a node on the given transmission path has a specific capacity $C_{j}$, we should take into account:

$$
\sum_{i} S_{P_{i}} \leq C,
$$

where $S_{P_{i}}$ is the value of the flow on the $i$ - th path entering the given node.

Going further, we should remember limited resources of the output interfaces:

$$
\sum_{i} S_{P_{i}}=\sum_{i} N_{P_{i}} \leq C_{j}^{k},
$$

where: $C_{j}^{k}$ represents the capacity of the $k$ - th interface on the $j$ - th node.

Let us refer to $[\mathbf{9}, \mathbf{1 0}]$, where the general logistic equation is defined for the whole family of systems:

$$
X=r N\left(1-\frac{N}{K}\right)^{f}=X_{M}(1-u)^{f},
$$

where $u=\frac{N}{K}, r$ is the constant factor of amplification, and $f$ the parameter of selforganization of a complex heterogeneous system.

Assuming that the whole transmission system, as well as each element (node) is itself a complex system we should accept the definition of the parameter $f$ proposed in $[\mathbf{9}, \mathbf{1 0}]$, and expressed by:

$$
f=\frac{\log \left(\frac{1}{1 \pm \beta r}\right)}{\log (1-u)} .
$$

Each node in the computer network is characterized by feedback $[\mathbf{9}, \mathbf{1 0}]$ resulting from the limited resources $K$. These limits apply to the node system resources, as well as they result from the relationship between the number of flows that can leave the interface, and flows incoming to it (2). In that case, assuming $N_{s}$ as the number of communication tasks that can not be handled, and $N_{e}$ as the number of communication tasks efficiently handled, we obtain the relationship: $N \pm N_{s}-N_{e}=0$.

Assuming that $\beta=\frac{N_{s}}{X}$ is the factor of the overload (rejection) of the packets, we get: $N_{e}=N \pm N_{s}=N \pm \beta X=N \pm \beta r N=N(1 \pm \beta r)$.

So far we have assumed the idealized performance model of the routing nodes based on the performance of a simple system defined by Malthus [11]: $X_{M}=r N$.

However, such an approach, in which we do not take into account the feedback depending on the parameter of self-organization, does not allow to use fully the self organisation of complex systems. Therefore, we should apply the following: 


$$
X=r N\left(1-\frac{N}{K}\right)^{\frac{\log \left(\frac{1}{1 \pm \beta r}\right)}{\log \left(1-\frac{N}{K}\right)}}=r K u(1-u)^{\frac{\log \left(\frac{1}{1 \pm \beta r}\right)}{\log (1-u)}} .
$$

On this basis we can define the performance of the node for the routing path.

$$
X_{P_{i}}=r N_{P_{i}}\left(1-\frac{N_{P_{i}}}{K_{P_{i}}^{j}}\right)^{\frac{\log \left(\frac{1}{1 \pm \beta r}\right)}{\log \left(1-\frac{N_{P_{i}}}{K_{P_{i}}^{j}}\right)}},
$$

where $N_{P_{i}}$ means the communication tasks on $i$ - th path, and $K_{P_{i}}^{j}$ - the resources for the $i$ - th path at the $j$ - th node. However, $\beta$ refers to the entire system. Thus, in the case of a particular communication path, $\beta$ must be associated with output interfaces for the path. So, $\beta_{P_{i}}^{l}$ is a feedback on the $i$ - th path in the $l$ - th node.

$$
X_{P_{i}}=r N_{P_{i}}\left(1-\frac{N_{P_{i}}}{K_{P_{i}}^{j}}\right)^{\frac{\log \left(\frac{1}{1 \pm \beta_{p_{i}}^{l}}\right)}{\log \left(1-\frac{N_{P_{i}}}{K_{P_{i}}^{j}}\right)}}=r K_{P_{i}}^{j} u(1-u)^{\frac{\log \left(\frac{1}{1 \pm \beta_{P_{i}}^{l}}\right)}{\log (1-u)}} .
$$

It is obvious that the increase in transmission efficiency while maintaining the constancy of communication resources is proportional to the degradation of their availability. From this perspective, analyzing the rate of change $\frac{d X_{P_{i}}}{d N_{P_{i}}}[\mathbf{1 0}]$, it is possible to assess the degradation (the use) of communication resources. Taking into account the fact that the full use of communication resources is revealed as a rejection of packets that can not be handled, you should modify the information transmission route.

In order to ensure the coexistence of the proposed solution with already existing routing protocols we should use ECMP functionality by using the proposed procedure new_local_route. This procedure checks whether there are alternative paths of the information routing, that have not been taken into account so far due to the fact that their cost or weight was worse than costs or weight of the preferred track (tracks). If they exist, then one of them will become an additional path of the transmission. Of course, the primary criterion for the selection of the path from a set of alternatives is the parameter (cost, weight) characteristic of a given routing protocol. Thus, the path with the best parameters will be selected first. An agent implemented on the node will modify the path cost to the value providing its share of the ECMP.

In the case when the node does not have any alternative path, the agent operating on the node must inform the relevant nodes about the situation. To do this, follow the procedurenode_search().

Let us introduce the following markings: $V_{P_{i}}=\left\{v_{P_{i}}^{-1}\right\}$ - the set of adjacent (previous) nodes on the $i$ - th path; $S_{P_{i}}^{l}$ - the flow on the $i$ - th path received in the $l$ - th interface. Within the procedure node_search(v), the following actions are implemented:

- analyse the previous nodes $\left.V=\left\{v_{i}^{(}-1\right)\right\}$, for which the node is the next jump on the path of transmission

- select a node $v_{i}^{-1}$, which generates the biggest traffic $\max \left(S_{P_{i}}^{l}\right)$. 
- inform the agent on the node $v_{i}^{-1}$ about the need to choose an additional, alternative route.

- implement the new_local_route $\left(v_{i}^{-1}\right)$ procedure at the agent located in the node $v_{i}^{-1}$.

- if the procedure new_local_route fails, start - on the node $v_{i}^{-1}$ - the node_search procedure.

Due to the fact that the agent acts on the node continuously, you must also specify the conditions for cancellation of an alternative path. For this purpose the agent analyses $X_{P_{i}}$. If its value drops below a determined value $\mu$, then the procedure del_path of an alternate route removal from the set of ECMP starts. This should be done as it should be remembered that this is a route of worse parameters of the transmission. Del_path procedure is initiated by the agent on the node $v_{i}$, which has started a new_local_route procedure.

Procedure dèl_path:

- If the alternative route added to ECMP is on the node $v_{i}$ the change its cost to its initial value (removal from the routing table).

- If the alternative route added to ECMP is not in the node $v_{i}$, then follow the procedure del_path on the node $v_{i}^{-1}$.

Del_path procedure works recursively at each particular node of the path. To avoid a selection of the route with the parameters significantly deviating from those of preferred routes, it should be possible to determine a threshold of acceptability when selecting an alternative route.

\section{Conclusions}

Currently used routing mechanisms, as well as ECMP, base on deterministic values of transmission parameters. Choosing a new route usually takes place only on the basis of the loss of the preferred route. Usually a change of the quality parameters of communication links is only fiction because currently implemented wired links are characterized by nearly $100 \%$ reliability and zero error rate.

The solution proposed in this paper allows to predict possible degradation of communication system resources, thus to make changes in the routing process for continuous match seeking. The adaptive system presented here, based on local agents, minimizes delays in comparison with the alternative centralized control system.

\section{References}

[1] Jong-Moon C., Analysis of MPLS Traffic Engineering, Circuits and Systems, 2000. Proceedings of the 43rd IEEE Midwest Symposium on , IEEE 2(5) (2000): 550.

[2] Srivastava S., Krithikaivasan B., Venkatachalam V., Beard C., Medhi D., A. van de Liefvoort, W. Alanqar, A. Nagarajan, A Case Study on Evaluating the Benefits of MPLS Traffic Engineering 
through Constraint-Based Routing and Network Controls, Communications, 2002, ICC 2002, IEEE International Conference 4 (2002): 2437.

[3] Fenglin L., Jianxun C, MPLS Traffic Engineering Load Balance Algorithm Using Deviation Path, Computer Science \& Service System (CSSS), 2012 International Conference, IEEE (2012): 601.

[4] Duangkreu W., Kerddit S., Noppanakeepong S., Frame relay to ATM PVC network interworking management, TENCON 2000, Proceedings IEEE, 1 (2000): 522.

[5] Sivabalan M., Mouftah H. T., Design considerations for link-state routing protocols, Computers and Communications, 1998. ISCC '98. Proceedings, Third IEEE Symposium, IEEE (1998): 53.

[6] Rivera R., Crichigno J., Ghani N., A Comparative Study of Routing Metrics for Reliable MultiPath Provisioni, 2014 International Conference on Computing, Networking and Communications, Network Algorithm \& Performance Evaluation Symposium, IEEE (2014): 450.

[7] Gouda, M. G., Schneider, M., Maximizable routing metrics, Networking, IEEE/ACM Transactions, IEEE 11(4) (2003): 663.

[8] Xu W., Yan P., Xia D., Wu M., Load Balancing Based on Similarity Multi-paths Routing, Parallel and Distributed Processing and Applications 3758 (2005): 345.

[9] Grabowski F., Nonextensive model of self-organizing systems, Complexity, Wiley 8(5) (2013): 28.

[10] Grabowski F., Logistic equation of arbitrary order, Physica A, Statistical Mechanics and its Applications 389 (2010): 3081.

[11] Malthus T. R., An essay on the Principle of Population, Pickering, London (1798). 